\title{
THE MATHEMATICAL MODELING OF ENTRAINED BIOLOGICAL OSCILLATORS $\dagger$
}

\author{
- J.GRASMAN \\ Centre for Mathematics and Computer Science, \\ Kruislaan 413, \\ 1098 SJ Amsterdam, The Netherlands
}

\begin{abstract}
In this paper perturbation methods are used for the mathematical analysis of coupled relaxation oscillators. This study covers entrainment by an external periodic stimulus as well as mutual entrainment of coupled oscillators with different limit cycles. The oscillators are of a type one meets in the modeling of biological oscillators by chemical reactions and electronic circuits. Special attention is given to entrainment different from $1: 1$. The results relate to phenomena occurring in physiological experiments, such as the periodic stimulation of neural and cardiac cells, and in the non-regular functioning of organs and organisms, such as the AV-block in the heart.
\end{abstract}

1. Introduction. Periodicity and synchrony play important roles in the temporal organization of activity in an organism. At the cellular level there is synchronization of neural and cardiac oscillators by cyclic inputs, as well as mutual synchronization (Holden, 1976; Ypey et al., 1980; Guevara et al., 1981; Cohen et al., 1982). At a higher level organs can be caused to follow the rhythm of an external pacemaker (Petrillo et al., 1983). Finally, the organism as a whole exhibits periodic activity known as the circadian rhythm: the rest-activity cycle of about $24 \mathrm{hr}$, which is entrained by the external light-dark cycle. In all these examples we primarily think of $1: 1$ entrainment. There is much literature on the mathematical modeling of this phenomenon; see Glass and Mackey (1979), Grasman and Jansen (1979) and Winfree (1980). However, entrainment with a frequency ratio different from $1: 1$ is also observed at all three of the levels of organization mentioned above. Cardiac muscle tissue may oscillate with a period that is a multiple of the forcing period (Guevara et al., 1981; Ypey et al., 1980). A heart may function in such a way that the contraction period of the ventricles and that of the atria have a ratio different from 1:1 (AV-block); see Keener (1981). In experiments one is able to entrain respiratory cycles of the lungs to the phase of a mechanical ventilator in a ratio different from 1:1 (Petrillo et al., 1983). The rest-activity rhythm of humans driven by the light-dark cycle can also be different from the 1:1 ratio. For infants

$\dagger$ Supported in part by the Science and Engineering Research Council of the United Kingdom under grant GR/C 26958. 
it may run $2: 1$ or higher. It is reported that such a synchrony is already present for the embryo driven by the mother's rhythm; see Gear Luce (1971). Moreover, some humans, isolated from external dark-light cycles, exhibit a 2:1 phase locking between body temperature and the rest-activity cycle (Wever, 1979). Compared with 1:1 entrainment, there are fewer studies on the mathematical modeling of $n: m$ entrainment for highly nonlinear oscillators; we mention Ermentrout (1981) and Glass and Perez (1982). Subharmonic entrainment by unidirectional coupling has been investigated by Yoshizawa et al. (1982). They found that the ordering of the ratios $n: m$ is related to Farey-type series in number theory. This aspect is studied in more detail by Allen (1983).

In this paper we analyze a system of $n$ coupled relaxation oscillators with intrinsic frequencies close to a ratio $j_{1}: j_{2}: \ldots: j_{n}$ with $j_{i}$ as integers. In our analysis we use singular and regular perturbation methods. The relaxation oscillator we consider is a Van der Pol-type differential equation with a small parameter $\epsilon$ multiplying the second derivative. This makes the system of coupled equations singularly perturbed. A second parameter $\delta$ is a measure for the deviation of the intrinsic frequencies from the ratio $j_{1}: j_{2}: \ldots: j_{n}$. Entrainment is possible if the coupling is at least of the same order of magnitude as the deviation. In Grasman and Jansen (1979) the case of weakly coupled, almost identical relaxation oscillators was analyzed and it was proved that the asymptotic solution indeed approximates an exact synchronized solution of the system. This proof, based on the work of Mishenko and Rosov (1980), also applies to the present configuration of coupled, non-identical oscillators. It is remarked that much of the mathematical results for 1:1 entrainment of almost identical oscillators carries over to $n: m$ entrainment. There is, however, one unexpected exception: in the case of periodic forcing of an oscillator with a smaller period the solution depends critically upon $\epsilon$, as appears in a numerical integration of the system for different $\epsilon$. The dependence is such that above a small value of $\epsilon$ the entrainment breaks down. This critical dependence also affects mutual entrainment. From the point of view of modeling entrained biological oscillators we obtain a better understanding of phenomena such as propagating phase waves, peaks in spectra of oscillatory systems and the phase fixation of an oscillator as a form of $n: m$ entrainment with $m=\infty$.

In Section 2 the discontinuous asymptotic approximation of a free relaxation oscillator is given. Furthermore, we consider the case where a periodic forcing term with an amplitude of order $0(\delta)$ is added to the equation. The forcing is of a type that does not change the limit cycle of the oscillator in the limit $\epsilon \rightarrow 0$. In this way only the phase of the oscillator is influenced in the asymptotic approximation. Let $T$ be the period of the 
driving force. Then we consider the mapping of the phase at time $t$ to the one at time $t=T$. For the case of piecewise linear relaxation oscillators one can compute this mapping explicitly. A stable fixed point of this mapping corresponds with an entrained solution. Without any difficulty this method can be extended to coupled oscillators; see Section 3 .

In Section 4 we study configurations of oscillators with diffusion coupling. In three examples we explore the possible relation with synchronization phenomena in biological systems. The first example deals with $n: m$ entrainment of two oscillators. The phenomenon of sudden cardiac death from a critical stimulus (Winfree, 1983) is described as a special case of an AVblock. In the second example the stability of propagating contraction waves in the gastrointestinal tract is discussed. In the final example we explore the spectrum of a densely coupled system of oscillators with widely different autonomous frequencies. This configuration exhibits a form of partial synchronization that is also found in populations of neural oscillators.

In Section 5 we deal with chemical and electronic oscillators that are frequently used for modeling biological oscillations. It is shown that they belong to the class of relaxation oscillators analyzed in this paper.

2. Free and Forced Oscillators. The relaxation oscillators we consider are of the type

$$
\begin{aligned}
\epsilon \mathrm{d} x / \mathrm{d} t & =y-F(x), \\
\mathrm{d} y / \mathrm{d} t & =a-x,
\end{aligned}
$$

where $\epsilon$ is a small positive parameter and $F$ a continuous, piecewise differentiable function satisfying $F(x) \rightarrow \pm \infty$ as $x \rightarrow \pm \infty$ and with one local maximum and minimum (see Figure 1a). Typical examples are the Van der Pol equation with $F(x)=\frac{1}{3} x^{3}-x(a=0)$ and piecewise linear differential equations (see Figure 2).

In Section 5 we deal with applications in chemistry and electronic networks, where $F$ follows, respectively, from the reaction dynamics and the diode characteristic. In this paper we concentrate on discontinuous approximations of periodic solutions of (2.1) as $\epsilon \rightarrow 0$. In Figure 1(a) we sketch the corresponding closed trajectory in the phase plane. The time-dependence of the $x$-component is given in Figure 1(b). The approximate solution over the two branches $A B$ and $C D$ satisfies

$$
F^{\prime}\left(X_{0}\right) \mathrm{d} X_{0} / \mathrm{d} t=-X_{0} .
$$

For the Van der Pol oscillator this equation can be integrated, giving an implicit expression for $X_{0}$ as a function of $t$. For the piecewise linear oscillator of Figure 2(a) the approximate solution has period $T_{0}=2 \ln 3$ and reads 


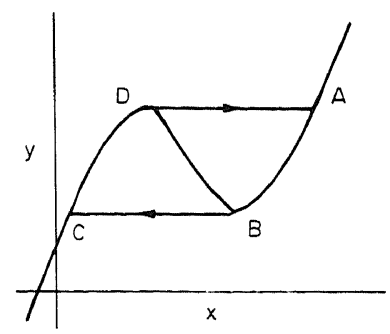

(a)

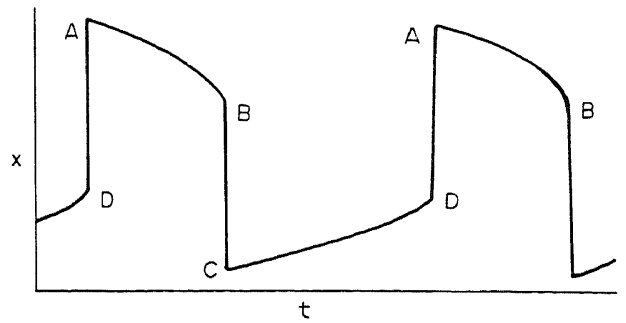

(b)

Figure 1. (a) The limit cycle in the phase plane as $\epsilon \rightarrow 0$. (b) The time dependence of the $x$-component of the limit solution.

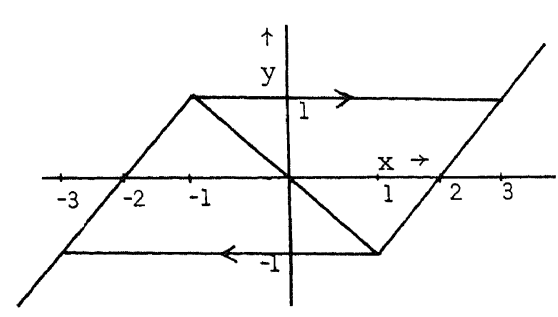

(a)

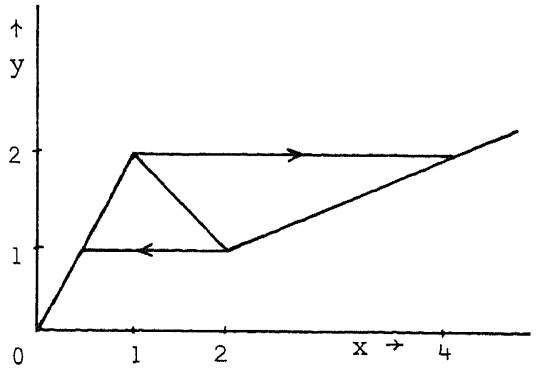

(b)

Figure 2. Piecewise linear oscillators. (a) $a=0$. (b) $a=1.5$.

$$
\begin{aligned}
& X_{0}(t)=3 \mathrm{e}^{-t} \text { for } 0<t<\ln 3, \\
& X_{0}(t)=\mathrm{e}^{-t} \text { for }-\ln 3<t<0, \\
& Y_{0}(t)=F\left(X_{0}(t)\right) .
\end{aligned}
$$

For differentiable functions $F$ the stable periodic solution of (2.1) is a limit cycle $\left(X_{\epsilon}, Y_{\epsilon}\right)$ which approaches $\left(X_{0}, Y_{0}\right)$ as $\epsilon \rightarrow 0$ and the period satisfies $T_{\epsilon}=T_{0}+0\left(\epsilon^{2 / 3}\right)$. For a proof of this we refer to Mishenko and Rosov (1980). Stoker (1950) states that for the piecewise linear oscillator $T_{\epsilon}=$ $T_{0}+0(\epsilon \ln \epsilon)$.

Next we take into consideration the periodic forcing of the relaxation oscillator (2.1) through its $y$-component:

$$
\begin{aligned}
\epsilon \mathrm{d} x / \mathrm{d} t & =y-F(x), \\
\mathrm{d} y / \mathrm{d} t & =a-x+\delta h(t), \quad h(t+T)=h(t),
\end{aligned}
$$

with $0<\epsilon \ll \delta \ll 1$ and $h(t)$ a piecewise continuous function. For $\epsilon \rightarrow 0$ 
the trajectories satisfy $y=F(x)$ or $y$ is constant, so that the forcing term $h$ will not change the closed trajectory in the phase plane. It may only influence the velocity of the oscillator on the limit cycle. Consequently, a solution of (2.4) is approximated by

$$
x=X_{0}(\phi(t)), \quad y=Y_{0}(\phi(t))
$$

where $\left(X_{0}(t), Y_{0}(t)\right)$ represents a discontinuous approximation of the free oscillator; see (2.3). Substitution in (2.4) for $\epsilon=0$ yields

$$
\frac{\mathrm{d} Y_{0}}{\mathrm{~d} \phi} \frac{\mathrm{d} \phi}{\mathrm{d} t}=a-X_{0}(\phi(t))+\delta h(t)
$$

or, using (2.2),

$$
\frac{\mathrm{d} \phi}{\mathrm{d} t}=1+\frac{\delta h(t)}{a-X_{0}(\phi(t))}, \quad \phi(0)=\alpha^{(0)}
$$

Integration gives the following approximation valid for bounded $t$ :

$$
\phi(t)=\alpha^{(0)}+t+\delta \int_{0}^{t} \frac{h(\bar{t})}{a-X_{0}\left(\alpha^{(0)}+\bar{t}\right)} \mathrm{d} \bar{t}+0\left(\delta^{2}\right) .
$$

Over one period $T$ the forcing causes a phase shift $\delta \psi\left(\alpha^{(0)}\right)$ with

$$
\psi(\alpha)=\int_{0}^{T} \frac{h(t)}{a-X_{0}(\alpha+t)} \mathrm{d} t .
$$

Considering the value of $\phi$ at times $t=k T$, we obtain the iteration map $P$ for the phase $\alpha^{(k+1)}=P \alpha^{(k)}$ or in a explicit form with accuracy $0\left(\delta^{2}\right)$ :

$$
\alpha^{(k+1)}=\alpha^{(k)}+T+\delta \psi\left(\alpha^{(k)}\right)(\bmod ) T_{0} .
$$

From the iteration map we analyze the limit behavior of the system. In the simplest case it has a stable fixed point that corresponds with a periodic solution of period $T$. Other possibilities are higher stable subharmonic solutions (see Figure 3c) and chaotic solutions for $\delta=0(1)$ (see Guckenheimer, 1980). Clearly a fixed point $\bar{\alpha}$ satisfies

$$
\psi(\bar{\alpha})=\left(m T_{0}-T\right) / \delta
$$

for some integer $m$ and is stable if $\psi^{\prime}(\bar{\alpha})<0$. Phase locking will occur if

$$
\min _{\alpha} \delta \psi(\alpha)<m T_{0}-T<\max _{\alpha} \delta \psi(\alpha) \text {. }
$$

3. Coupled Oscillators. We are now in the position to handle systems of coupled relaxation oscillators satisfying 


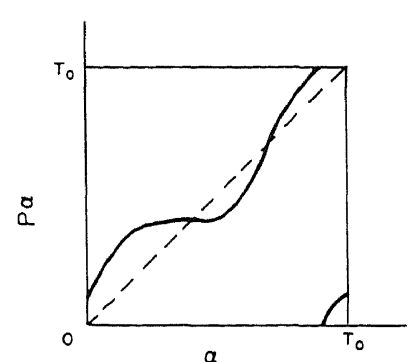

(a)

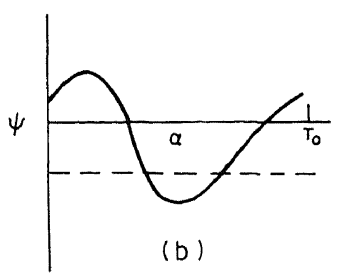

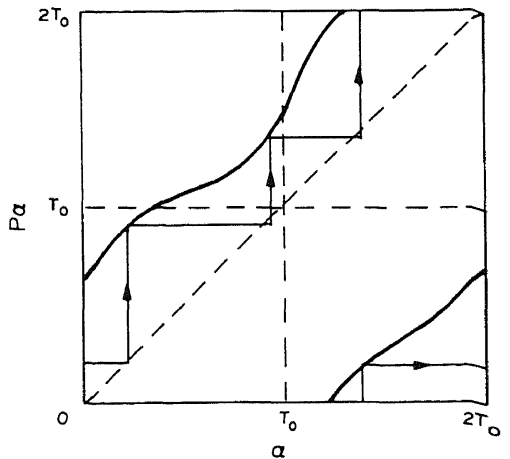

(c)

Figure 3. (a) The iteration map $P$. (b) The phase shift function $\psi$. (c) A higher order fixed point of the map $P$.

$$
\begin{aligned}
\epsilon \mathrm{d} x_{i} / \mathrm{d} t & =y_{i}-F_{i}\left(x_{i}\right) \\
\mathrm{d} y_{i} / \mathrm{d} t & =c_{i}(\delta)\left(a_{i}-x_{i}\right)+\delta \sum_{j \neq i} H_{i j}\left(x_{i}, y_{i}, x_{j}, y_{j}\right),
\end{aligned}
$$

where $H_{i j}$ is assumed to be continuous. Each oscillator describes a free oscillation given by $\left(X_{i 0}\left(\phi_{i 0}(t)\right), Y_{i 0}\left(\phi_{i 0}(t)\right)\right)$, with

$$
\phi_{i 0}(t)=\alpha_{i}^{(0)}+c_{i}(\delta) t .
$$

In case the oscillators are coupled, the phase functions are approximated by

$$
\phi_{i}(t)=\phi_{i 0}(t)+\delta \sum_{j \neq i} \int_{0}^{t} \frac{H_{i j}\left(\phi_{i 0}(\bar{t}), \phi_{j 0}(\bar{t})\right)}{a_{i}-X_{i}\left(\phi_{i 0}(\bar{t})\right)} \mathrm{d} \bar{t}+0\left(\delta^{2}\right) .
$$

Let us assume that the unperturbed oscillators $(\delta=0)$ have autonomous periods $T_{i \epsilon}$ satisfying

$$
T_{10}: T_{20}: \ldots: T_{n 0}=j_{1}: j_{2}: \ldots: j_{n},
$$

where $j_{i}, i=1, \ldots, n$ are integers. The fact that $c_{i}(\delta)=c_{i}(0)+0(\delta)$ results in autonomous periods of the perturbed system that make $0(\delta)$ differ from this ratio. Next we introduce the common unperturbed period $T$, the smallest number for which the quotients $T / T_{i 0}, i=1, \ldots, n$ are positive integers. 
The phase shift function is defined by

$$
\psi_{i j}\left(\alpha_{i}, \alpha_{j}\right)=\int_{0}^{T} \frac{H_{i j}\left(\alpha_{i}+c_{i}(\delta) t, \alpha_{j}+c_{j}(\delta) t\right)}{X_{i}\left(\alpha_{i}+c_{i}(\delta) t\right)} \mathrm{d} t, \quad i \neq j .
$$

For the iteration map $P$ of the phases $\alpha_{i}^{(k)}$ at times $t=k T$ we obtain

$$
\alpha_{i}^{(k+1)}=\alpha_{i}^{(k)}+c_{i}(\delta) T+\delta \sum_{j \neq i} \psi_{i j}\left(\alpha_{i}^{(k)}, \alpha_{j}^{(k)}\right)(\bmod ) T_{0}
$$

for $i=1, \ldots, n$ or $\alpha^{(k+1)}=P \alpha^{(k)}$. More specifically, the phase shift function $\psi_{i j}$ depends upon $\beta_{i j}=\alpha_{i}-\alpha_{j}$, as seen from (3.5) by shifting the integration interval over $\alpha_{j}$. If we set $\alpha_{1}=0$, then all phase differences $\beta_{i j}$ are uniquely determined from the remaining $n-1$ phases $\alpha_{j}$. The system (3.1) has a periodic solution with a period of about $T$ if the following system of $n$ algebraic equations for $\alpha_{2}, \ldots, \alpha_{n}$ and $q$ has a solution:

$$
c_{i}(\delta) T+\delta \sum_{j \neq 1} \psi_{i j}\left(\beta_{i j}\right)=\delta q(\bmod ) T_{0}, \quad i=1, \ldots, n .
$$

The period of the approximation for $\epsilon \rightarrow 0$ takes the value $T+\delta q$.

Example. As an example we deal with two coupled oscillators, which for $\epsilon \rightarrow 0$ have the same limit cycles in the phase plane and with autonomous frequencies that differ by about factor 3 . We take a type of coupling that the simplifies the computations:

$$
\begin{aligned}
& \epsilon \mathrm{d} x_{1} / \mathrm{d} t=y_{1}-F\left(x_{1}\right), \quad \mathrm{d} x_{2} / \mathrm{d} t=y_{2}-F\left(x_{2}\right), \\
& \mathrm{d} y_{1} / \mathrm{d} t=-(1-\delta d) x_{1}+\delta a_{1} x_{2}, \quad \mathrm{~d} y_{2} / \mathrm{d} t=-3 x_{2}+\delta a_{2} x_{1},
\end{aligned}
$$

with $F(x)$ as given by Figure 2(a). Carrying out the computations set out in the foregoing section, we arrive at the phase shift functions $\psi_{12}$ and $\psi_{21}$ satisfying

$$
\begin{aligned}
a_{1} \psi_{21}(\beta) & =a_{2} \psi_{12}(-\beta), \quad \beta=\alpha_{1}-\alpha_{2}, \\
\psi_{12}(\beta) & =a_{1}\left\{\mathrm{e}^{\beta}(-4 / 3-\gamma)+4 / 3 \mathrm{e}^{3 \beta}\right\} \text { for } 0 \leqslant \beta<1 / 3 \ln 3,
\end{aligned}
$$

with $\gamma=3^{-1 / 3}+3^{2 / 3}-3^{1 / 3}-3^{2 / 3}$. For $1 / 3 \ln 3 \leqslant \beta \leqslant 0$ we have

$$
\psi_{i j}(\beta)=\psi_{i j}(\beta+1 / 3 \ln 3) \text {. }
$$

Let us compare these asymptotic results for $\epsilon=0$ with numerical solutions of 3.8 for fixed small parameter values $\left(\epsilon=10^{-3}\right.$ and $\left.\delta=0.25\right)$. In Figure $4(\mathrm{a})$ we present the result for $\left(a_{1}, a_{2}\right)=(1,0)$. It is observed that the values of the entrained numerical solutions $(d, \beta(\epsilon))$ are close to the stable branch of the phase shift function $\psi_{12}(\beta)$. The value $\beta(\epsilon)$ is found as the difference in time at the successive intersections of $x_{1}(t)$ and $x_{2}(t)$ with 


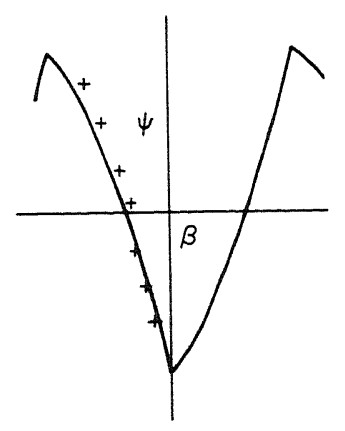

(a)

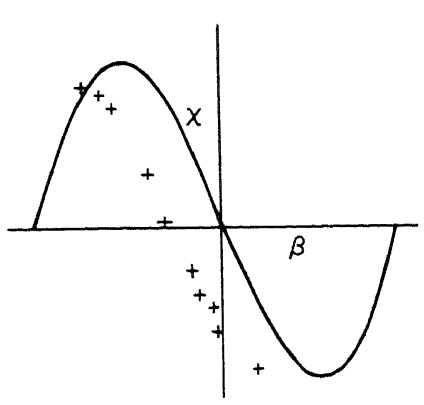

(c)

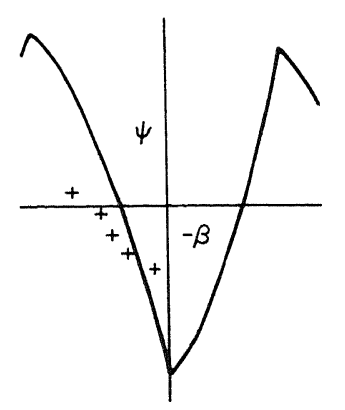

(b)

Figure 4. Numerical ( ++$)$ and asymptotic solutions of (3.8). (a) $\left(a_{1}, a_{2}\right)=$ $(1,0)$. (b) $\left(a_{1}, a_{2}\right)=(0,1)$. (c) $\left(a_{1}, a_{2}\right)=(1,1)$.

the line $x=0$. For the case $\left(a_{1}, a_{2}\right)=(0,1)$ the outcome is quite different (see Figure 4(b)). The phase shift curve turns out to be very sensitive to the value of $\epsilon$. For $\epsilon=0.002$ the bandwidth of entrainment is reduced by a factor of 2; this occurs again at $\epsilon=0.004$. At $\epsilon=0.005$ entrainment virtually breaks down. Finally, in Figure 4(c) we sketch the result of mutually entrained numerical solutions, $\left(a_{1}, a_{2}\right)=(1,1)$. The values $(d, \beta(\epsilon))$ for the numerical solutions are away from the stable branch of the relative phase shift function $\chi(\beta)=\psi_{12}(\beta)-\psi_{21}(-\beta)$. A further comparison shows that the outcome of the numerical solutions is consistent with the results for $\left(a_{1}, a_{2}\right)=(0,1),(1,0)$.

4. Diffusion Coupling in Biological Systems. From a biological point of view it is worthwhile concentrating on a specific type of coupling. Let us assume that the two components $x$ and $y$ represent a biochemical reactant which may diffuse from one compartment of the biological system to the other. When diffusion of the component $x$ can be neglected we arrive at 
a system of type (3.1), with

$$
H_{i j}\left(x_{i}, y_{i}, x_{j}, y_{j}\right)=p\left(y_{j}-y_{i}\right), \quad i \neq j, \quad p>0 .
$$

We analyze the dynamics of such a system of coupled oscillators for diffeent types of configurations. The following three choices have been made for a system of oscillators with identical limit cycles (see Figure $2 b$ ) and widely different autonomous frequencies. As in the example given in Section 3, analytical expressions for the phase shift functions can be computed. However, this is quite laborious and not necessary for obtaining quantitative results on entrainment.

$n: m$ entrainment. We consider two mutually coupled oscillators with $c_{1}(\delta)=1$ and $c_{2}(\delta)=c$. In Figure 5 we give the domains in the $c, p$-plane where the algebraic equation (3.7) has a solution for $T=T_{0}, 2 T_{0}, 3 T_{0}$, $4 T_{0}, 6 T_{0}$ and $12 T_{0}$. These solutions come in pairs (a stable and an unstable one). Each domain corresponds with an entrained solution of synchronized period ratio $n: m$. Note that the domains overlap and that oscillator 2 can be fixated in two different phases for $c$ small.

This configuration of two coupled oscillators can be seen as a model for $n: m$ entrainment in biological systems, as we mentioned in the introduction; see also Ermentrout (1981).

Winfree (1983) and Van Meerwijk et al. (1983) investigate a mechanism by which an oscillator stops after receiving a stimulus at a critical phase of its cycle. From the present model we conclude that an oscillator can also be stopped by a non-critical periodic stimulus. The problematic point in the critical stimulus mechanism is the instability of the phaseless set in the state space. Van Meerwijk et al. (1983) observed a tendency of the system to return to the stable oscillatory state; Winfree postulated the presence of a black hole at that spot. In the present model the stopping of a coupled oscillator is a stable mechanism, occurring in two different states. Compared with other entrained solutions at those parameter values they may have a small domain attraction, so that also in this case a critical stimulus may be needed. In terms of cardiac arrythmias, it can be seen as an $\mathrm{AV}$-block with ratio $1: \infty$.

This phenomenon has also been noticed by Ypey et al. (1982). In their study the periodic stimulus needed to have a high frequency. Presumably, the domain of attraction of the driven oscillatory state then vanishes and the state of phase fixation takes over.

$A$ chain of oscillators with decreasing autonomous frequency. It is assumed that for $n$ oscillators equation (4.1) is satisfied for $j=i \pm 1,1 \leqslant j \leqslant n$ and that $H_{i j}=0$ in all other cases. Moreover, we set

$$
c_{i}(\delta)=c_{0}+i \Delta c, \quad i=1, \ldots, n .
$$




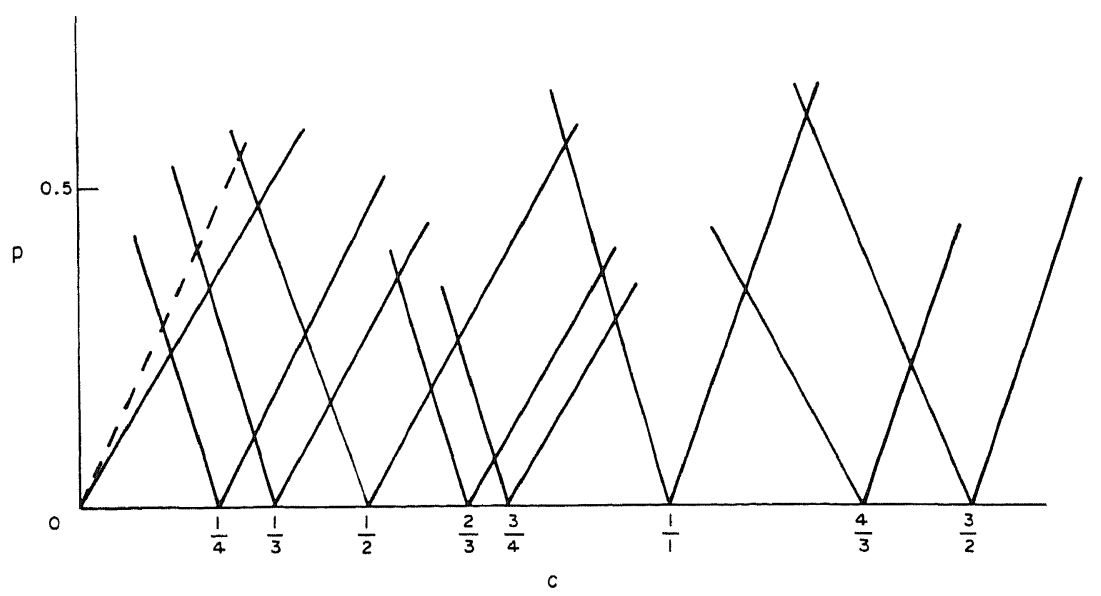

Figure 5. Domains of $n: m$ entrainment.

Instead of trying to solve the algebraic equation (3.7) with $T$ necessarily very large, we compute numerically the iteration map $P$ for $T=T_{0}$ and carry out a number of iterations until a stable pattern of actual phases and phase velocities arises. The phase velocities are averaged over the last 12 iterations. In the simulation the pattern is independent of the choice of initial phases. About $n$ iterations are needed to arrive at such a pattern. Depending on the gradient of $c_{i}$, we observe the formation of compartments of oscillators with equal phase velocities (see Figure 6). For a small gradient the compartments, also called plateaus, are large (see Ermentrout and Kopell, 1983). In the extreme case we have one compartment being the complete chain in a fully synchronized state. For larger gradients the compartments shrink and increase in number. The size of the compartments is not equally distributed over the chain. In the present situation the one at the slow end dominates. In Grasman and Jansen (1979), where a different type of coupling is analyzed, the one at the fast end dominates. The present study differs from the ones mentioned above at the point where widely different autonomous frequencies are allowed. In this way we include the effect of large gradients in $c_{i}$. From simulations in this range we found that, although the compartments were shrinking and synchrony decreased, the pattern of phase waves, running from the fast to the slow end, persisted. From the point of view of biological applications, this is an important observation. The above configuration can be seen as a model for the gastrointestinal tract. In this system the same type of distribution of autonomous frequencies of contraction is present. It is concluded that the gradient may be quite large to guarantee the desired (direction of) propagation without risking a breakdown of the wave pattern because of decreasing synchrony; see also Sarna et al. (1972). 


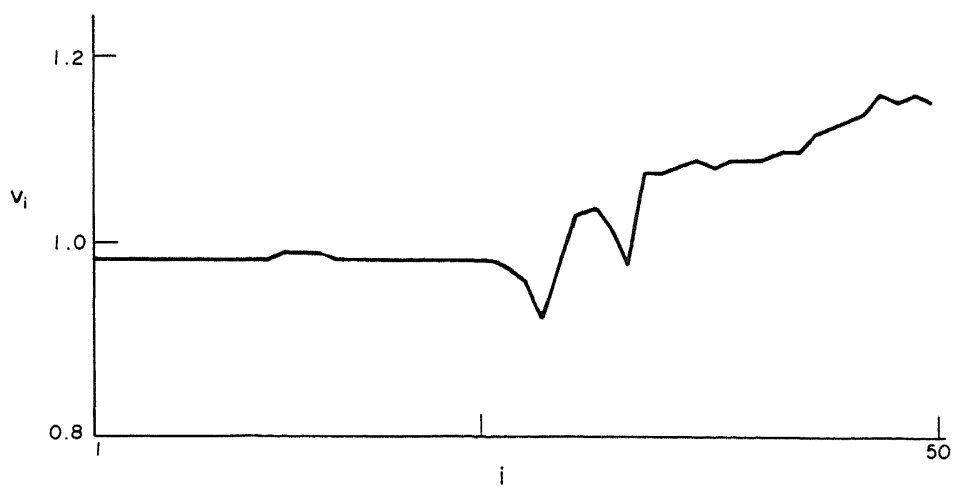

(a)

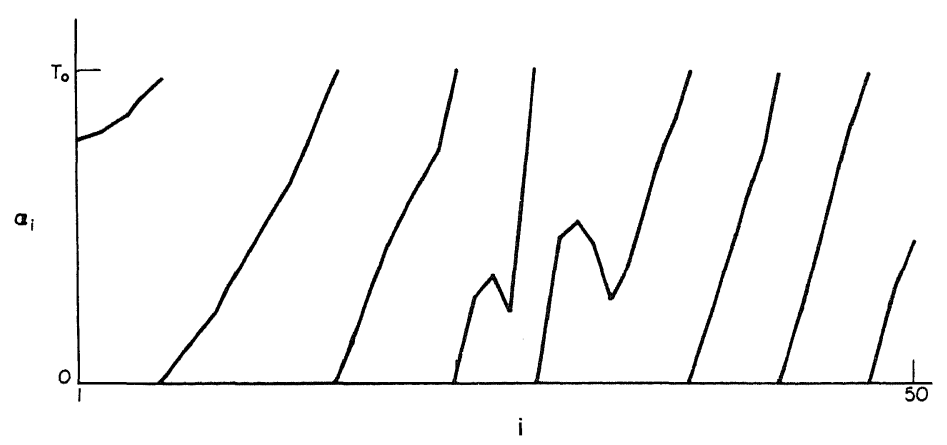

(b)

Figure 6. Entrainment in a chain of oscillators satisfying (4.2) with $n=50$, $\delta=0.3, c_{0}=1.9$ and $\Delta c=0.004$ after 156 iterations of the map $P$. (a) The phase velocity. (b) The phase.

Large populations of coupled oscillators with widely different frequencies. Next we consider a system of $n$ oscillators, all mutually coupled, as given by (4.1). They are assumed to have autonomous phase velocities that are uniformly distributed over the $c_{i}$-interval $(0.5,1.5)$. Again we analyze the iteration map $P$ with the phase velocities averaged over the last 12 iterations. As seen in Figure 7, the spectrum of phase velocities exhibits peaks spaced in such a way that their relative positions have a ratio $n: m$. Other oscillators move forwards and backwards over the spectrum without locking in at any of these peaks. There is some analogy with neural oscillators forming a densely coupled system. In theoretical studies of the EEG it is postulated that peaks are due to the mechanism of entrainment. Wiener (1958) speculates that a combination of three peaks could be explained from entrainment of oscillators with nearby frequencies (the central peak). 


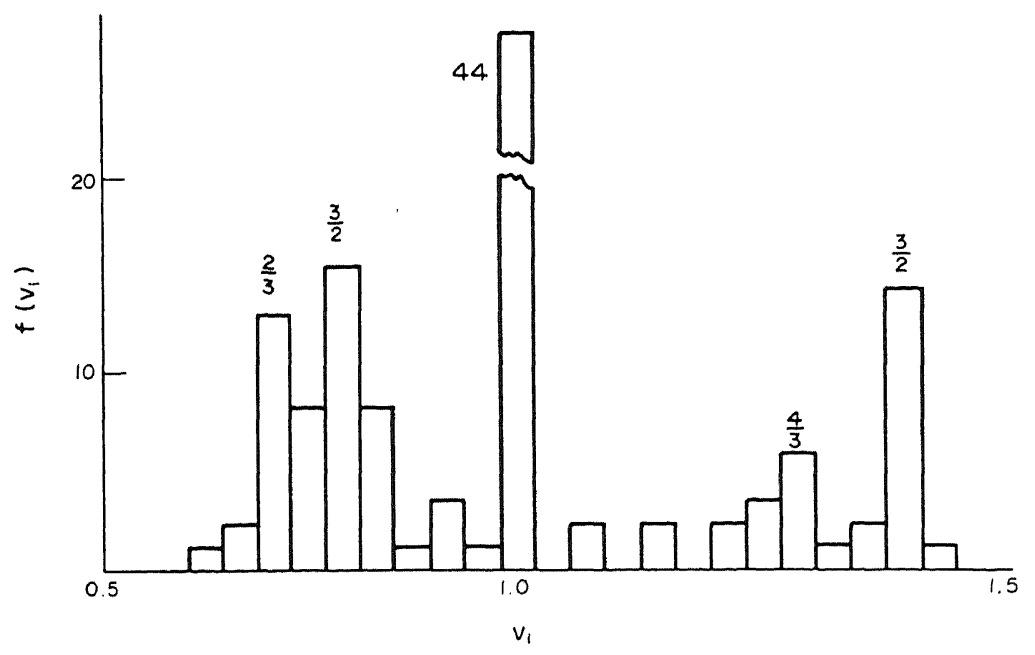

Figure 7. Distribution of phase velocities in a population of 100 mutually coupled oscillators with intrinsic phase velocity homogeneously distributed over the interval $(0.5,1.5)$.

The two side-peaks consist of oscillators with frequencies too far away to get entrained. This idea has been elaborated by Kreifeldt (1970) and Kuramoto (1975). Our numerical simulations suggest that peaks may also occur as a result of $n: m$ entrainment in cases where the oscillators have different frequencies. Lopes da Silva et al. (1976) observe such peaks in their model of an interacting neural population.

5. Applications in the Theory of Chemical and Electronic Oscillations. The physiology of periodic phenomena in organisms can be quite complex, and in most cases is not understood in sufficient detail. In the process of investigation one uses prototypes of biological oscillators in order to gain more insight in the mechanism of entrainment and related phenomena. Besides abstract mathematical models there are prototypes of oscillators orginating from inorganic chemistry, e.g. the Belousov-Zhabotinskii reaction (Tyson, 1976), and from electronic circuit theory (Van der Pol and Van der Mark, 1928; Hoppensteadt, 1981). In this section we show that two such models can be cast in the form of relaxation oscillators of the type we study in this paper.

First we consider a hypothetical chemical reaction with periodic fluctuations in the concentration of some of the reactants: the Bruxellator (see Auchmuty and Nicolis, 1976). Schematically we have the following reaction: 


$$
\begin{gathered}
A \stackrel{k_{1}}{\underset{k_{-1}}{\rightleftharpoons}} X, \\
B+X \underset{k_{-2}}{\stackrel{k_{2}}{\rightleftharpoons}} Y+D, \\
2 X+Y \underset{k_{-3}}{\stackrel{k_{3}}{\rightleftharpoons}} 3 X, \\
X \underset{k_{-4}}{\stackrel{k_{4}}{\rightleftharpoons}} E .
\end{gathered}
$$

Seeping the reactants $A, B, D$ and $E$ at a constant level and setting the everse reactions all zero, we obtain for the concentration of $X$ and the um of $X$ and $Y$ the following system of differential equations in dimensioness variables:

$$
\begin{aligned}
& \mathrm{d} u / \mathrm{d} \tau=1-u-\beta u+\alpha u^{2}(w-u)=\beta f(u, w ; \beta), \\
& \mathrm{d} w / \mathrm{d} \tau=1-u=g(u, w) .
\end{aligned}
$$

This system has the equilibrium point $(\bar{u}, \bar{w})=(1,1+\beta / \alpha)$, which is stable for $\beta<1+\alpha$. Varying $\beta$, we find that the equilibrium point is unstable tbove the critical value $\beta_{c}=1+\alpha$. Then a stable limit cycle with amplitude $\left(\beta-\beta_{c}\right)^{\frac{1}{2}}$ branches off. For $\beta>\alpha+1 \gg 1$ with $\beta-\alpha=0(1)$ the limit zycle turns into a relaxation oscillation (see Figure 8 ). The two stable branches depend in this case on the large parameter. They approximately satisfy

$$
w_{1}(u)=\frac{\beta u-1}{\alpha u^{2}} \text { and } w_{2}(u)=u+\frac{\beta}{\alpha u} .
$$

The contributions to the period from these branches are

$$
\int_{a_{i}}^{b_{i}} \frac{1}{1-u} \frac{\mathrm{d} w_{i}}{\mathrm{~d} u} \mathrm{~d} u, \quad i=1,2
$$

with

$$
a_{1}=\alpha / \beta^{2}, b_{1}=1 / \beta, a_{2}=\beta^{2} / 4 \alpha \text { and } b_{2}=\sqrt{ } \beta / \alpha .
$$

Consequently, we find for the period of the autonomous Bruxellator

$$
T_{0}=\frac{\beta^{2}}{4 \alpha}-\frac{\beta^{3}}{\alpha^{2}}+\frac{\beta^{4}}{\alpha^{3}}+\ln \beta-\ln \alpha+0(1) .
$$

For the analysis of weakly coupled Bruxellators we may proceed as in 


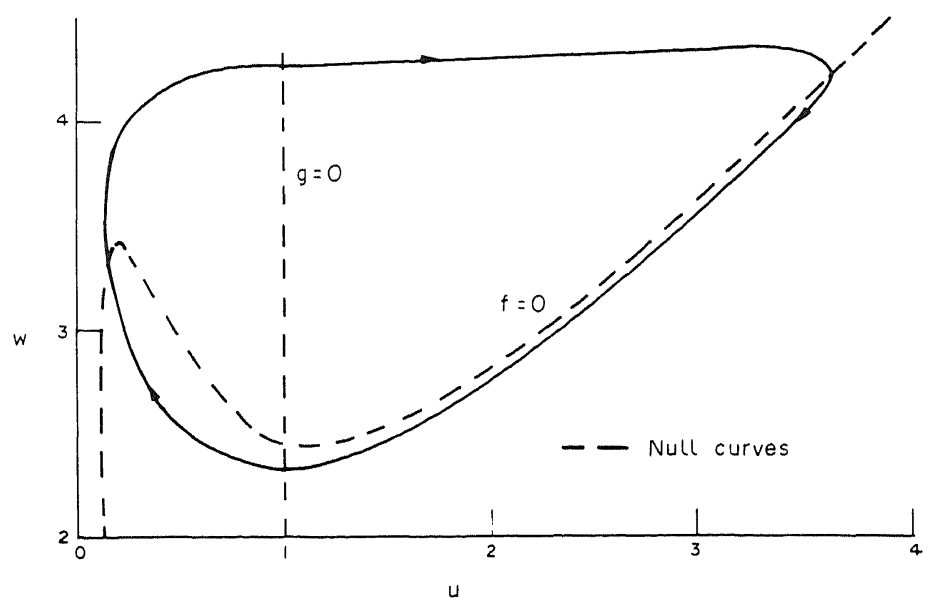

Figure 8. Limit cycle of the Bruxellator for $\alpha=5$ and $\beta=7$.

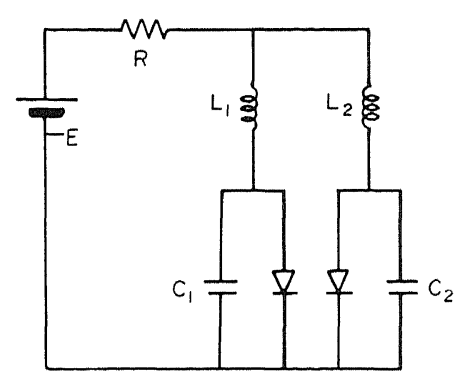

(a)

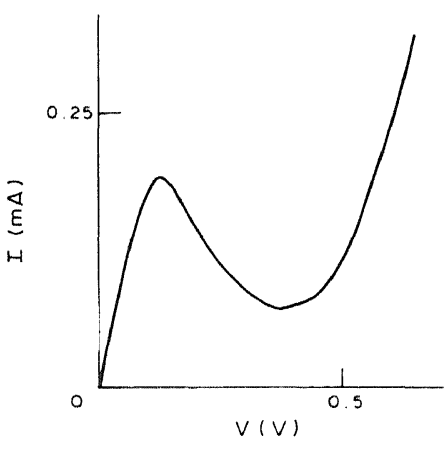

(b)

Figure 9. Oscillations in an electronic network with two tunnel diodes. (a) The circuit. (b) The characteristic of the tunnel diode.

Sections 3 and 4. In Grasman and Jansen (1979) such a system of relaxation oscillators was analyzed with the asymptotic method of Section 3 and gave rise to bulk oscillations and stable phase wave patterns. These regular oscillatory patterns agree qualitatively with numerical results by Auchmuty and Nicolis (1976) for Bruxellators with diffusion coupling. There are also other approaches to the mathematical analysis of coupled chemical oscillators; we mention Torre (1975) and Neu (1980).

Finally, we discuss the occurrence of entrained oscillations in an electronic circuit. Gollub et al. (1978) analyzed the circuit given in Figure 9(a). The two tunnel diodes have characteristics as sketched in Figure 9(b). For this circuit the voltage and current satisfy the system of differential equations: 


$$
\begin{array}{ll}
Y_{1} \mathrm{~d} V_{1} / \mathrm{d} t=I_{1}-F\left(V_{1}\right), & C_{2} \mathrm{~d} V_{2} / \mathrm{d} t=I_{2}-F\left(V_{2}\right), \\
L_{1} \mathrm{~d} I_{1} / \mathrm{d} t=E-V_{1}-R\left(I_{1}+I_{2}\right), & L_{2} \mathrm{~d} I_{2} / \mathrm{d} t=E-V_{2}-R\left(I_{1}+I_{2}\right) .
\end{array}
$$

ior $R$ small and $C_{1}$ and $C_{2}$ of even smaller orders of magnitude this system $s$ of the type we studied with asymptotic methods. In Gollub et al. (1978) ntrainment phenomena are observed similar to that of piecewise linear sscillators.

\section{LITERATURE}

lllen, T. 1983. "On the Arithmetic of Phase Locking: Coupled Neurons as a Lattice on $\mathrm{R}^{2}$." Physica 6D, 305-320.

tuchmuty, J. F. G. and G. Nicolis, 1976. "Bifurcation Analysis of Reaction Diffusion Equations-III. Chemical Oscillations." Bull. math. Biol. 38, 325-350.

Zohen, A. H., P. J. Holmes and R. H. Rand. 1982. "The Nature of the Coupling between Segmental Oscillators of the Lamprey Spinal Generator for Locomotion: a Mathematical Model." J. math. Biol. 13, 345-369.

Ermentrout, G. B. 1981. " $n: m$ Phase-locking of Weakly Coupled Oscillators." J. math. Biol. 12, 327-342.

- and N. Kopell. 1983. "Frequency Plateaus in a Chain of Weakly Coupled Oscillators I and II." Boston, MA: Department of Mathematics, Northeastern University, preprints.

Jear Luce, C. 1971. Biological Rhythms in Human and Animal Physiology. New York: Dover.

Jlass, L. and M. C. Mackey. 1979. "A Simple Model for Phase Locking of Biological Oscillators." J. math. Biol. 7, 339-352.

- and R. Perez. 1982. "Fine Structure of Phase Locking." Phys. Rev. Lett. 48, $1772-1775$.

Gollub, J. P., T. O. Brunner and B. C. Danly. 1978. "Periodicity and Chaos in Coupled Nonlinear Oscillators." Science 200, 48-50.

Grasman, J. and M. J. W. Jansen. 1979. "Mutually Synchronized Relaxation Oscillators as Prototypes of Oscillating Systems in Biology." J. math. Biol. 7, 171-197.

Guckenheimer, J. 1980. "Symbolic Dynamics and Relaxation Oscillations." Physica $1 \mathrm{D}, 227-235$.

Guevara, M. R., L. Glass and A. Shrier. 1981. "Phase Locking, Period-doubling Bifurcations and Irregular Dynamics in Periodically Stimulated Cardiac Cells." Science 214, 1350-1353.

Holden, A. V. 1976. "The Response of Excitable Membrane Models to a Cyclic Input." Biol. Cybernet. 21, 1-7.

Hoppensteadt, F. C. 1981. "Electric Models of Neurons." Lect. appl. Math. 19, 327-344.

Keener, J. P. 1981. "On Cardiac Arrythmias: AV Conduction Block." J. math. Biol. $12,215-225$

Kreifeldt. J. 1970. "Ensemble Entrainment of Self-sustaining Oscillators: a Possible Application to Neural Signals." Math. Biosci. 8, 425-436.

Kuramoto, Y. 1975. "Self Entrainment of a Population of Coupled Nonlinear Oscillators." In Lecture Notes in Physics, No. 39. International Symposium on Mathematical Prob lems in Theoretical Physics, Ed. H. Araki, pp. 420-422. Berlin: Springer.

Lopes da Silva, F. H., A. van Rotterdam, P. Barts, E. van Heusden and W. Burr. 1976. "Models of Neuronal Populations: the Basic Mechanisms of Rhythmicity." In Progress in Brain Research, Ed. D. Swaab and M. E. Corner, Vol. 45, pp. 281-308. Amsterdam: Biomedical Press. 
Mishenko, E. F. and N. Kh. Rosov. 1980. Differential Equations with Small Parameters and Relaxation Oscillations. New York: Plenum Press.

Neu, J. C. 1980. "Large Populations of Coupled Chemical Oscillators." SIAM J. appl. Math. 38, 305-316.

Petrillo, G. A., L. Glass and T. Trippenbach. 1983. "Phase Locking of the Respiratory Rhythm in Cats to a Mechanical Ventilator." Can. J. Physiol. Pharmacol. 61, 599-607.

Sarna, S. K., E. E. Daniel and Y. J. Kingma. 1972. "Simulation of the Electric-control Activity of the Stomach by an Array of Oscillators." Digest. Dis. 17, 299-310.

Stoker, J. J. 1950. Nonlinear Vibrations. New York: Interscience.

Torre, V. 1975. "Synchronization on Nonlinear Biochemical Oscillators Coupled by Diffusion." Biol. Cybernet. 17, 137-144.

Tyson, J. J. 1976. Lecture Notes in Biomathematics, Vol. 10. The Belousov-Zhabotinskii Reaction. Berlin: Springer.

Van der Pol, B. and J. Van der Mark. 1928. "The Heart Beat Considered as a Relaxation Oscillation and an Electrical model of the Heart." Phil. Mag. 4, 763-773.

Van Meerwijk, W. P. M., G. de Bruin, A. C. G. van Ginneken, J. van Hartevelt, H. J. Jongsma, S. S. Scott and D. L. Ypey. 1983. "Phase Resetting Properties of Cardiac Pacemaker Cells." Amsterdam: Department of Physiology, University of Amsterdam, preprint.

Wever, R. A. 1979. The Circadian System of Man. New York: Springer.

Wiener, N. 1958. Nonlinear Problems in Random Theory. Cambridge, MA: MIT Press.

Winfree, A. T. 1980. "The Geometry of Biological Time." Biomathematics Vol. 8. Berlin, Springer.

-. 1983. "Sudden Cardiac Death: a Problem in Topology." Sci. Am. 248, 118-131.

Yoshizawa, S., H. Osada and J. Nagumo. 1982. "Pulse Sequences Generated by a Degenerate Analog Neuron Model." Biol. Cybernet. 45, 23-34.

Ypey, D. L., W. P. M. van Meerdijk, E. Ince and G. Groos. 1980. "Mutual Entrainment of Two Pacemaker Cells. A Study with an Electronic Parallel Conductance Model." J. theor. Biol. 86, 731-755.

- - and G. de Bruin. 1982. "Suppression of Pacemaker Activity by Rapid Repetitive Phase Delay." Biol. Cybernet. 45, 187-194.

RECEIVED 2-8-83

REVISED 10-31-83 